\title{
Predispositional genome sequencing in healthy adults: design, participant characteristics, and early outcomes of the PeopleSeq Consortium
}

Emilie S. Zoltick ${ }^{1,2+}$, Michael D. Linderman ${ }^{3,4+}$, Molly A. McGinniss 5 , Erica Ramos ${ }^{6}$, Madeleine P. Ball7, George M. Church ${ }^{7,8,9,10}$, Debra G. B. Leonard ${ }^{11}$, Stacey Pereira ${ }^{12}$, Amy L. McGuire ${ }^{12}$, C. Thomas Caskey ${ }^{13}$, Saskia C. Sanderson ${ }^{4,14}$, Eric E. Schadt ${ }^{4}$, Daiva E. Nielsen ${ }^{1,15}$, Scott D. Crawford ${ }^{16}$, Robert C. Green ${ }^{1,17,18,19^{*}}$ and for the PeopleSeq Consortium

\begin{abstract}
Background: Increasing numbers of healthy individuals are undergoing predispositional personal genome sequencing. Here we describe the design and early outcomes of the PeopleSeq Consortium, a multi-cohort collaboration of predispositional genome sequencing projects, which is examining the medical, behavioral, and economic outcomes of returning genomic sequencing information to healthy individuals.
\end{abstract}

Methods: Apparently healthy adults who participated in four of the sequencing projects in the Consortium were included. Web-based surveys were administered before and after genomic results disclosure, or in some cases only after results disclosure. Surveys inquired about sociodemographic characteristics, motivations and concerns, behavioral and medical responses to sequencing results, and perceived utility.

Results: Among 1395 eligible individuals, 658 enrolled in the Consortium when contacted and 543 have completed a survey after receiving their genomic results thus far (mean age 53.0 years, $61.4 \%$ male, $91.7 \%$ white, $95.5 \%$ college graduates). Most participants (98.1\%) were motivated to undergo sequencing because of curiosity about their genetic make-up. The most commonly reported concerns prior to pursuing sequencing included how well the results would predict future risk (59.2\%) and the complexity of genetic variant interpretation (56.8\%), while $47.8 \%$ of participants were concerned about the privacy of their genetic information. Half of participants reported discussing their genomic results with a healthcare provider during a median of 8.0 months after receiving the results; 13.5\% reported making an additional appointment with a healthcare provider specifically because of their results. Few participants $(<10 \%)$ reported making changes to their diet, exercise habits, or insurance coverage because of their results. Many participants (39.5\%) reported learning something new to improve their health that they did not know before. Reporting regret or harm from the decision to undergo sequencing was rare $(<3.0 \%)$.

\footnotetext{
* Correspondence: rcgreen@bwh.harvard.edu

${ }^{\dagger}$ Emilie S. Zoltick and Michael D. Linderman contributed equally to this work.

'Division of Genetics, Department of Medicine, Brigham and Women's

Hospital, 41 Avenue Louis Pasteur, Suite 301, Boston, MA 02115, USA

${ }^{17}$ The Broad Institute of MIT and Harvard, 415 Main Street, Cambridge, MA

02142, USA

Full list of author information is available at the end of the article
}

(c) The Author(s). 2019 Open Access This article is distributed under the terms of the Creative Commons Attribution 4.0 International License (http://creativecommons.org/licenses/by/4.0/), which permits unrestricted use, distribution, and reproduction in any medium, provided you give appropriate credit to the original author(s) and the source, provide a link to the Creative Commons license, and indicate if changes were made. The Creative Commons Public Domain Dedication waiver (http://creativecommons.org/publicdomain/zero/1.0/) applies to the data made available in this article, unless otherwise stated. 
(Continued from previous page)

Conclusions: Healthy individuals who underwent predispositional sequencing expressed some concern around privacy prior to pursuing sequencing, but were enthusiastic about their experience and not distressed by their results. While reporting value in their health-related results, few participants reported making medical or lifestyle changes.

Keywords: Personal genome sequencing, Return of results, Genomics, Test utility, Public health

\section{Background}

Whole genome and exome sequencing have well-established clinical utility for rare disease diagnosis [1, 2] and personalized cancer treatment [3]. Driven by decreasing costs, sequencing is increasingly being used in other clinical, research, and commercial settings, including as a screening tool in apparently healthy individuals, termed predispositional personal genome sequencing (PPGS) [4]. Individuals pursuing PPGS are not using genome sequencing for diagnostic purposes, but nonetheless are interested in obtaining health-related results. For many participants and providers, there is an expectation that PPGS will eventually enable a more personalized and preventive approach to medicine, in which illness is anticipated or prevented through screening for genetic predispositions to disease [5]. However, the actual clinical utility in healthy individuals is unknown, and the risks, benefits, and costs both to the individual and society are unclear $[6,7]$. Despite this uncertainty, there are increasing numbers of PPGS projects underway, both research- and industry-based [4].

Unlike screening tests that detect early signs of disease, PPGS screens for a potential predisposition to disease. Of particular concern are individuals who will be falsely identified as being at risk $[6,7]$ as a result of analytic errors, interpretation errors, and/or gaps in understanding of the penetrance of each variant, especially in the absence of family history of the condition [8]. These factors may present challenges for clinical management, which in turn, could create unnecessary anxiety and medical surveillance, and drive increased healthcare expenditures [9]. Previous and ongoing PPGS studies have reported numerous examples of successful predispositional identification of individuals with disease or increased disease risk $[10,11]$. Recent reports show that as many as $20 \%$ of participants in predispositional sequencing cohorts may have a variant with monogenic disease risk [12-14]. At this early stage, however, the precise fraction of individuals who might benefit from sequencing due to disease prevention or early diagnosis is uncertain.

Multiple research studies have sought to improve our understanding of the possible clinical and personal utility of PPGS. The Harvard Personal Genome Project (PGP), launched in 2005, was arguably the first PPGS project, though the return of genomic results and the consequences thereof were not the main purpose [15].
More recent studies, conducted mostly as controlled research protocols in clinical settings, have explored the reactions to sequencing and the return of results to healthy individuals [11, 16-23]. For example, within Geisinger, an integrated healthcare system, there are plans for over 250,000 patient-participants to have their exomes sequenced, most without a specific indication, under the MyCode ${ }^{\oplus}$ Community Health Initiative, an expansive clinical research protocol [21]. The All of Us Research Program, part of the Precision Medicine Initiative (PMI), has publicly committed to providing participants access to the data gathered, which might include genomic information, although the details have not yet been finalized [24]. Multiple biotech companies have launched or announced broad physician-ordered predispositional sequencing panels [25-28] and more consumer-oriented research products [29-32]. These developments reflect the multiple forces, such as changing views on obligations to return study results to participants, disintermediation of traditional medical authorities, and large-scale sequencing efforts seeking pharmaceutical targets, which are accelerating the availability of PPGS.

The Personal Genome Sequencing Outcomes (PeopleSeq) Consortium is a collaborative effort among multiple academic and commercial PPGS projects designed to cost-effectively collect coherent survey data on the shortand long-term outcomes of apparently healthy individuals already undergoing sequencing through one of the Consortium projects. Thus far, the Consortium has enrolled individuals across four PPGS projects who have received a broad range of health-related genomic results from personal genome or exome sequencing. Additional sites are in the process of joining the Consortium, representing a range of both research and commercial sequencing providers. This Consortium then provides a mechanism to examine the outcomes of PPGS in the many different contexts in which this technology is now employed. PeopleSeq participants are innovators and early adopters [33], similar to initial users of other rapidly evolving technologies. Studying PPGS early adopters can provide valuable insights because these individuals have actually used these technologies, and thus can provide concrete evidence of both the risks and benefits [34]. Here we describe the design of the PeopleSeq Consortium and present descriptive findings from the four initial projects. 


\section{Methods \\ Overview}

The PeopleSeq Consortium, formed in 2014, is a collaboration of PPGS projects designed to study participants' motivations and experiences and the self-reported medical, behavioral, and economic outcomes of PPGS. Here we report on data collected from participants in the first four projects to contribute to the Consortium: the Harvard PGP [15], the Baylor College of Medicine's Young Presidents' Organization (YPO) and $\mathrm{MD} / \mathrm{PhD}$ Genome Projects [17], Mt. Sinai's HealthSeq project [19], and Illumina's Understand Your Genome (UYG) program [35]. All of the projects performed genome or exome sequencing on apparently healthy adults and returned a report of the individual genomic results back to participants. The consent, genetic counseling, and results disclosure processes varied by project (see Additional file 1: Table S1 for an overview of each project and Linderman et al. [4] for a more detailed review of these and similar projects). Clinical genome reports from a CLIA (Clinical Laboratory Improvement Amendments)-certified laboratory were only provided by UYG. UYG was also the only project to send a clinical report to a healthcare provider, while PGP publicly shared participants' genomes online. Though the type of PPGS results varied by project, all of the projects returned monogenic disease findings in numerous genes (more than the genes specified in the American College of Genetics and Genomics recommendations for reporting of secondary findings) $[36,37]$. The PeopleSeq Consortium itself did not perform sequencing of participants but administered common web-based surveys to participants in the collaborating projects. Consent for participation to receive surveys from the PeopleSeq Consortium was entirely separate from consent in the original PPGS project.

Depending on the site and timing of the invitation, participants were invited to enroll and complete PeopleSeq surveys either before or after disclosure of their genomic results. Those who enrolled into the PeopleSeq Consortium after disclosure of their PPGS results received one initial survey (the "catch-up" survey), while those who enrolled before disclosure of their PPGS results received two surveys: a survey after signing up for PPGS but before disclosure of their PPGS results (the pre-disclosure survey) and after disclosure (the post-disclosure survey). At the Consortium's conception, all participants received the catch-up survey; however, since November 2015, the preand post-disclosure surveys were administered whenever possible. All participants received annual follow-up surveys. This longitudinal design allows for assessment of both short- and long-term outcomes. The Partners HealthCare Human Research Committee and the Baylor College of Medicine Institutional Review Board approved the entire study. Each site consulted their Institutional Review Board, as applicable, and received additional approval if necessary.

\section{Survey design and measures}

The PeopleSeq Consortium surveys were developed by an interdisciplinary team of geneticists, genetic counselors, ethicists, psychologists, and survey design researchers. The surveys were adapted from the Impact of Personal Genomics (PGen) Study surveys [38], developed using expert consultation and cognitive interview techniques, with additional questions derived from qualitative interviews in the MedSeq Project [18]. The survey questions assessed sociodemographic characteristics, personal and family health information, prior use of genetic testing, motivations and concerns, psychological impacts, risk perceptions, perceived utility and harms, and behavioral and medical responses (see Additional file 1: Table S2 for a summary of the survey measures and Additional file 2 for copies of the study surveys). Where possible, the surveys utilized validated measures of psychological states [39-41] and decision regret [42], as well as published scales for genomics self-efficacy [43], genome sequencing knowledge [44], and other measures [45].

Genome sequencing knowledge was evaluated using an 11-item assessment with 5-level Likert scale responses (strongly disagree to strongly agree) [44]. Responses for negatively worded items were reversed to make "strongly agree" the correct response for all items. Decision regret was measured with a 5-item instrument with a 5-level Likert scale used for agreement with each item (scored 1-5 points) [42]. The mean score across items was calculated and then converted to a total score out of 100 with higher scores indicating greater regret.

Some survey questions were tailored to each project to reflect differences in the participant experience. For example, questions were populated with site-specific names and some questions only included response options corresponding to the genomic results provided by that project. Following continued analysis of preliminary data, the surveys have been revised over time, refining the wording of some of the questions that were not part of validated measures and adding or deleting questions as necessary. While this refinement has improved comprehension and shortened the surveys, these changes have resulted in some missing data for early participants.

\section{Participant recruitment and data collection}

The PeopleSeq Consortium recruited adults aged 18 years or older who independently decided to pursue PPGS through one of the collaborating projects and, when required by the collaborating project, consented to be contacted about related research projects. All eligible individuals who were previously enrolled in PGP, HealthSeq, and the YPO and MD/PhD Genome Projects received an invitation to participate in the Consortium via email with a description of the study and a link directing them to the web-based survey. Potential participants 
from UYG were initially approached in person, by email, or via announcement at a UYG program event before receiving the PeopleSeq invitation email.

The invitation email to complete the catch-up survey was sent at least 2 months after receipt of PPGS results. For participants enrolled prior to their PPGS results disclosure, an email invitation to complete the pre-disclosure survey was sent 1 to 3 months before disclosure of genomic results, and the post-disclosure survey was sent 2 to 3 months after receipt of their genomic results. The timing of these emails was coordinated between the collaborating site and SoundRocket (Ann Arbor, MI). If a participant did not complete the pre-disclosure survey prior to receiving their PPGS results, an invitation to complete the catch-up survey was sent. If, at the start of the post-disclosure or catch-up survey, a participant indicated that they had not viewed their PPGS results, they were not permitted to complete the survey until they had done so. Participants were invited to complete an annual follow-up survey. To improve the response rate, each survey invitation email was followed by up to four reminder emails for nonresponders. Participants were also entered into a random drawing to win an Apple Watch.

Informed consent was obtained electronically before participants were given access to the initial study survey (either the pre-disclosure or catch-up survey). The surveys were administered on SoundRocket's secure web-based platform. Each participant was assigned a unique master identifier, which allowed the participant to securely access the survey system. Participants could freely navigate forward and backward through the survey. All items in the survey were optional and could be skipped. All responses were saved, allowing for partially completed surveys and permitting participants to complete the survey in multiple sessions. At the end of the post-disclosure and catch-up surveys, all participants (except for those from the YPO and $\mathrm{MD} / \mathrm{PhD}$ Genome Projects) were further asked to consent to share their genomic results with the Consortium. For those participants who consented to share their results, the study coordinated with each collaborating site to obtain this information. PGP participants were asked to provide their PGP identifier linked to their already publicly available genomic data.

\section{Data analyses}

The main analyses presented in this report were limited to the 543 participants who completed the catch-up survey or both the pre- and post-disclosure surveys and responded to the referenced question. A comparison of responders and nonresponders to the initial Consortium invitation was conducted in a substudy of 1093 individuals invited to participate from the two projects, PGP (352 invitees) and UYG (741 invitees), for which case-level nonresponder demographic data were available (data limited to age, gender, and race only). Chi-square tests were used to assess demographic differences by response status.

In the main analyses, for participants completing both the pre- and post-disclosure surveys, sociodemographic characteristics, motivations and concerns when deciding to pursue sequencing, and previous genetic testing were reported on the pre-disclosure survey while all other data were from the post-disclosure survey. Descriptive statistics including means with standard deviations and counts with percentages were computed for participant demographics, psychological outcomes, downstream behavioral and medical actions, perceived utility, and attitudes regarding genome sequencing. Differences in sociodemographic characteristics by project were compared by ANOVA for continuous variables and chi-square or Fisher's exact test for categorical variables. Psychological, behavioral, and medical responses, perceived utility, and attitudes were compared by survey (pre-/post-disclosure surveys and catch-up survey), given the differences in timing of survey administration. To test differences by survey, the Wilcoxon rank sum test was used for continuous and ordinal measures and chi-square or Fisher's exact tests were used for categorical measures. Minimal statistically significant differences were observed by survey (Additional file 1: Tables S3 and S4), so the results are presented for all surveys combined. In an exploratory analysis, a sign test was used to compare perceived present and future personal or clinical utility of the genomic results. All statistical tests were two-sided with $p<0.05$ considered statistically significant. The data were analyzed using SAS software (version 9.4; SAS Institute, Cary, NC). The statistical code is available from the authors upon request.

\section{Results}

A total of 1395 eligible individuals from four projects were invited to participate in the PeopleSeq Consortium between October 2014 and July 2017 (see Fig. 1 for an

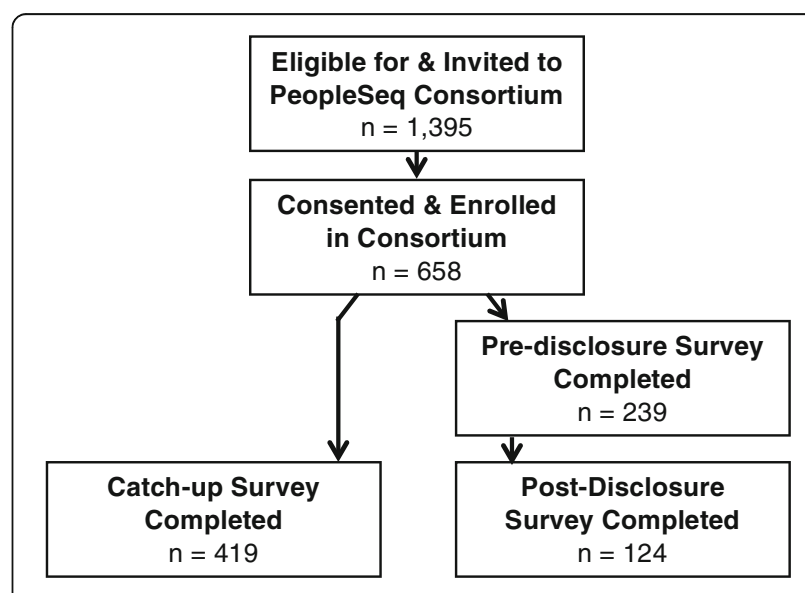

Fig. 1 PeopleSeq Consortium enrollment and data collection 
enrollment flowchart and Additional file 1: Figure S1 for a flowchart by project). A total of 658 individuals consented and enrolled in the study for an initial response rate of $47.2 \%$. A total of 543 participants completed a survey after receiving their genomic results (419 completed the catch-up survey and 124 completed both the pre- and post-disclosure surveys), for a response rate of $38.9 \%$. The post-disclosure survey is pending for 115 participants who either have received but not yet returned the survey or have not yet received their sequencing results.

In a substudy of responders and nonresponders from UYG and PGP in which case-level demographic nonresponder data were available, the initial response rate was $30.7 \%$. Females were overrepresented among the responders compared to nonresponders $(39.6 \%$ versus $32.2 \%$, respectively, $p=0.018$; see Additional file 1: Figure S2). There were borderline statistically significant differences in the distributions of race and age by response status in which a higher percentage of white and younger adults were responders, particularly for PGP.

The sociodemographic characteristics of the 543 participants who completed a survey after receiving their genomic results are summarized in Table 1 . Nearly two thirds of participants were male and most self-identified as white. Almost all participants had at least a college degree, and most participants had an annual household income of $\$ 100,000$ or greater. When asked about their occupation, $29.1 \%$ reported that they were healthcare professionals (including healthcare providers and clinical researchers). Almost all participants described themselves as having good health or better; only $4.0 \%$ self-reported fair or poor health. About half of participants reported receiving some form of personal genetic testing prior to undergoing PPGS for this project. More than $70 \%$ of these individuals reported using direct-to-consumer (DTC) genetic testing (genotyping rather than whole genome sequencing), and "curiosity about health and traits predicted by my genetic make-up" and ancestry were the most common reasons for using DTC genetic testing. While there were statistically significant differences in the sociodemographic characteristics across the four projects, participants in each of the projects were predominantly older, well-educated, wealthy, and in good health (Additional file 1: Table S5).

Figure 2 presents the proportion of participants endorsing each motivation for pursuing PPGS from a provided list. When asked what was the most important factor in deciding to pursue PPGS, "curiosity about my genetic make-up" and "interest in finding out about my personal disease risk" ranked highest with about $15 \%$ of participants endorsing each (data not shown). When provided with a list of possible concerns when deciding
Table 1 Characteristics of participants with completed postdisclosure or catch-up surveys in the PeopleSeq Consortium ( $n=543$ )

\begin{tabular}{|c|c|}
\hline Characteristic & No. $(\%)^{a}$ \\
\hline Age, mean ( \pm SD; range), years & $\begin{array}{l}53.0(12.8 \\
22-91)\end{array}$ \\
\hline \multicolumn{2}{|l|}{ Gender } \\
\hline Female & $202(38.0)$ \\
\hline Male & $326(61.4)$ \\
\hline Other & $3(0.6)$ \\
\hline \multicolumn{2}{|l|}{ Race } \\
\hline African American or Black & $3(0.6)$ \\
\hline Asian & $15(2.8)$ \\
\hline White & $485(91.7)$ \\
\hline More than one race or other race & $26(4.9)$ \\
\hline Hispanic or Latino & $16(3.0)$ \\
\hline \multicolumn{2}{|l|}{ Education } \\
\hline Less than college degree & $24(4.5)$ \\
\hline College degree & $67(12.7)$ \\
\hline Some graduate school & $160(30.3)$ \\
\hline Doctoral or professional degree & $278(52.6)$ \\
\hline \multicolumn{2}{|l|}{ Annual income } \\
\hline$<\$ 40,000$ & $36(7.0)$ \\
\hline$\$ 40,000-\$ 99,999$ & $82(15.9)$ \\
\hline$\geq \$ 100,000$ & $397(77.1)$ \\
\hline \multicolumn{2}{|l|}{ Marital status } \\
\hline Married & $383(72.0)$ \\
\hline Widowed, divorced, or separated & $71(13.4)$ \\
\hline Never married & $78(14.7)$ \\
\hline Biological children & $370(69.6)$ \\
\hline US resident & $469(88.3)$ \\
\hline \multicolumn{2}{|l|}{ Self-reported health } \\
\hline Excellent & $167(35.2)$ \\
\hline Very good & $210(44.3)$ \\
\hline Good & $78(16.5)$ \\
\hline Fair & $18(3.8)$ \\
\hline Poor & $1(0.2)$ \\
\hline Prior genetic testing & $247(49.8)$ \\
\hline \multicolumn{2}{|l|}{ Project } \\
\hline Illumina's Understand Your Genome & $329(60.6)$ \\
\hline The Harvard Personal Genome Project & $167(30.8)$ \\
\hline $\begin{array}{l}\text { Baylor Young Presidents' Organization and MD/PhD } \\
\text { Genome Projects }\end{array}$ & $28(5.2)$ \\
\hline Mount Sinai's HealthSeq project & $19(3.5)$ \\
\hline
\end{tabular}

$S D$ standard deviation

aPercentages may not sum to 100 due to rounding. Percentages and means are not all based on total of 543 participants because of missing responses to some survey items. The percent of missing responses ranges between 0 and $12.7 \%$ (median $=2.4 \%$ missing) 


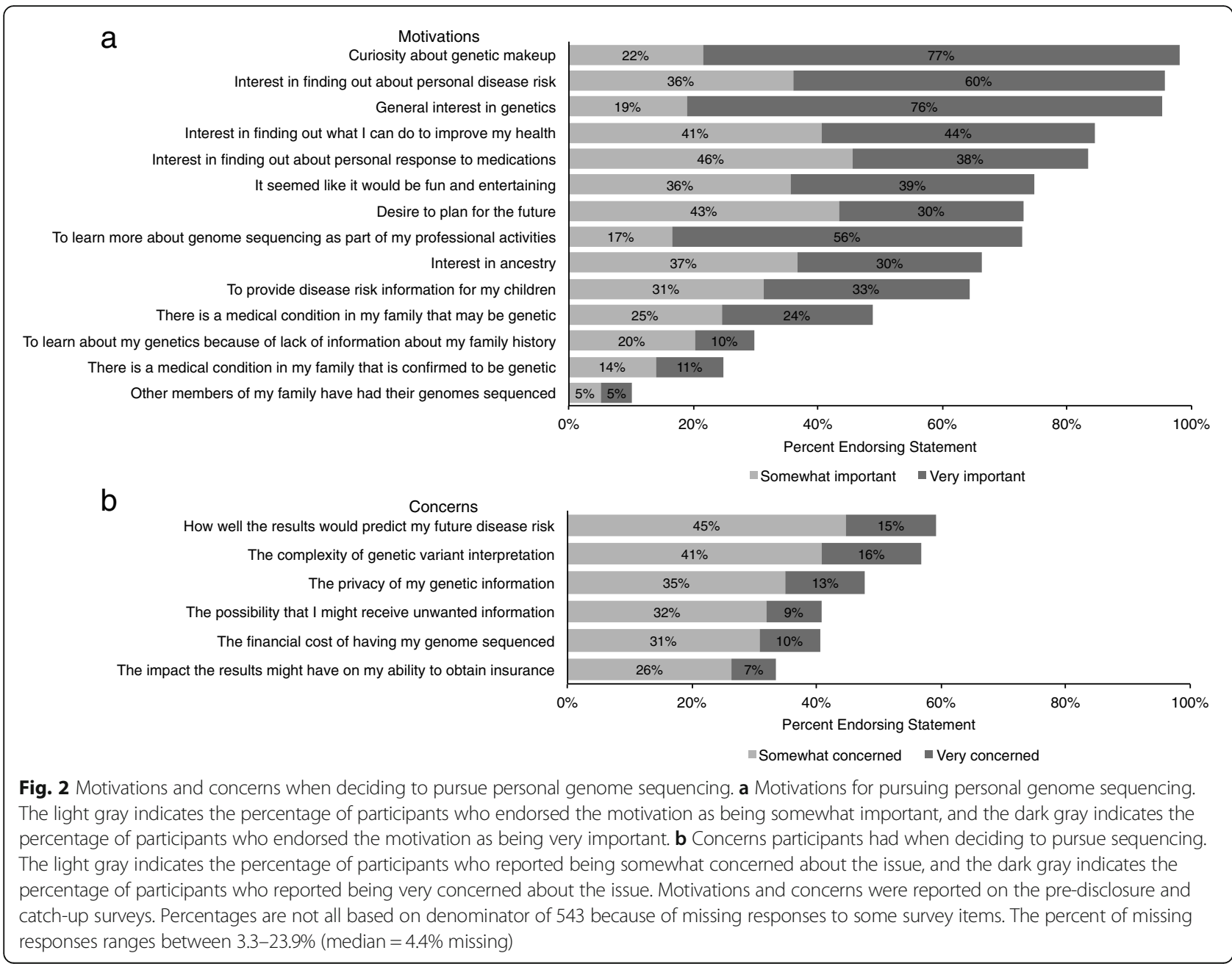

to pursue PPGS, "how well the results would predict my future risk" (59.2\%) and "the complexity of genetic variant interpretation" (56.8\%) were most frequently selected as factors that participants were somewhat or very concerned about (Fig. 2). Privacy of the genetic information was a concern for $47.8 \%$ of participants, but only $12.8 \%$ (range 2.5-22.2\% across projects) of participants were very concerned about privacy; this was lowest among participants sequenced through PGP and ranged from $16.8 \%$ for UYG to $22.2 \%$ for the HealthSeq project participants. Only $7.1 \%$ (range $3.1-11.1 \%$ across projects) of participants were very concerned, while $26.4 \%$ (range $18.9-44.4 \%$ across projects) were somewhat concerned, about the impact of the PPGS results on their ability to obtain insurance. Strong concerns regarding insurance discrimination were lowest among participants in PGP and highest among participants in the HealthSeq project.

The initial survey after PPGS results disclosure was completed a median of 8.0 months after disclosure (15.2 months after disclosure for participants completing the catch-up survey and 3.8 months for those completing the post-disclosure survey). During this time, most participants $(86.8 \%)$ reported discussing their results with someone; $81.1 \%$ of participants reported sharing their results with a family member (Table 2 ). Half $(51.2 \%)$ of participants reported discussing their PPGS results with a healthcare provider, although fewer reported that they had made (13.5\%; range $10.6-16.7 \%$ across projects) or planned to make (5.8\%; range $0-6.9 \%$ across projects) an additional appointment with a healthcare provider specifically because of their genomic results. Reporting discussion of the PPGS results with a provider was lowest among PGP participants (41.2\%) and highest among participants in the YPO and MD/PhD Genome Projects (79.2\%). Of those participants discussing their results with a provider, a primary care physician was the most frequently cited type of provider consulted (81.1\%), followed by a genetics specialist (27.9\%). The majority of these individuals $(81.8 \%)$ were somewhat or very satisfied with the discussion of their PPGS results with their healthcare provider, while $6.2 \%$ reported that their 
Table 2 Reported responses following disclosure of genome sequencing results

\begin{tabular}{|c|c|}
\hline & No. $(\%)^{a}$ \\
\hline \multicolumn{2}{|l|}{ Psychological response } \\
\hline Decision regret score, mean $( \pm S D \text {; range })^{b}$ & $6.6(13.4 ; 0-100)$ \\
\hline \multicolumn{2}{|l|}{ Behavioral and medical responses because of sequencing results } \\
\hline \multicolumn{2}{|l|}{ Communication of test results } \\
\hline Family & $399(81.1)$ \\
\hline Healthcare provider & $252(51.2)$ \\
\hline Made appointment with healthcare provider & $65(13.5)$ \\
\hline Sought out more information about health or medical topics related to results & $237(48.5)$ \\
\hline Made changes to diet & $45(9.4)$ \\
\hline Made changes to exercise routine & $41(8.6)$ \\
\hline Made changes to medications & $29(7.2)$ \\
\hline Made changes to insurance coverage & $2(0.4)$ \\
\hline
\end{tabular}

$S D$ standard deviation

a Percentages and means are not all based on total of 543 participants because of missing responses to some survey items. The percent of missing responses ranges between 8.7 and $25.8 \%$ (median $=11.0 \%$ missing)

$\mathrm{b}_{5}$-item decision regret scale provides a score from 0 to 100

healthcare provider was unwilling to discuss the meaning of their PPGS results. Dissatisfaction with the discussion of their results was highest for the PGP participants (12.9\% not at all satisfied) and lowest for participants from the HealthSeq project and YPO and $\mathrm{MD} / \mathrm{PhD}$ Genome Projects ( $0 \%$ not at all satisfied), with $6.5 \%$ of UYG participants not at all satisfied with the discussion. Due to their PPGS results, $12.5 \%$ (range $0-17.4 \%$ across projects) of participants reported that they had one or more tests, medical exams, or procedures, and it was only in the HealthSeq project that no one reported having any tests, medical exams, or procedures because of their PPGS results. Of those individuals reporting any tests, exams, or procedures, $13.3 \%$ reported undergoing one or more genetic tests to confirm their PPGS findings.

Few participants (12.4\%) reported making any lifestyle changes because of their PPGS results: $9.0 \%$ and $8.6 \%$ reported eating a healthier diet and exercising more, respectively, while less than $1.0 \%$ and $0 \%$ reported eating a less healthy diet and exercising less. Less than $1.0 \%$ of participants reported making changes to any of their insurance coverage including health, life, long-term care, and disability insurance.

Decision regret following PPGS results disclosure was rare with $60.3 \%$ of participants reporting no decision regret (score of $0 / 100$ ) and $95.0 \%$ of participants having a score of $25 / 100$ or less. Fewer than $3.0 \%$ of participants directly reported regretting their decision to pursue PPGS or experiencing harm due to this decision. When asked how valuable they felt the PPGS experience was, $88.5 \%$ reported that the experience was somewhat $(44.0 \%)$ or very $(44.5 \%)$ valuable. In ranking the perceived utility of their PPGS results on a scale from 1 to
10, participants believed that the information would be more useful in the future (median $=8.0$ ) than now (median $=6.0)$, a statistically significant difference $(P<$ 0.0001). More than one third of participants (39.5\%) somewhat or strongly agreed that they believed they learned something to improve their health that they did not know before, and $58.4 \%$ somewhat or strongly agreed that having PPGS made them feel like they had more control over their health (Table 3). However, more than half of participants $(54.6 \%)$ were disappointed that their PPGS results did not tell them more information. This was not for lack of understanding, as $79.3 \%$ of participants agreed that they felt confident that they understood their PPGS results, which corresponded with an 11-item genome sequencing knowledge assessment in which > $70 \%$ of participants answered 8 of the 11 items correctly. Almost all participants (96.2\%) were somewhat $(28.8 \%)$ or very $(67.4 \%)$ satisfied with their decision to obtain PPGS.

Following PPGS, 84.2\% of participants felt somewhat $(25.5 \%)$ or very $(58.7 \%)$ comfortable with the idea of sharing their entire genome sequence in general. As long as their identity remained anonymous, over $65 \%$ of participants across the projects would be willing to share their entire genome publicly. Most participants were also in agreement that personal genomic information should be part of the standard medical record $(79.9 \%)$ and that health insurance should cover PPGS (62.8\%) (Table 3). A minority of participants agreed that PPGS should only be available to people through their doctor $(26.6 \%)$. Almost a quarter of participants (23.4\%) both strongly agreed that personal genomic information should be part of the medical record and strongly disagreed that sequencing should only be available to people through their doctor. 
Table 3 Degree of agreement/disagreement on perceived utility and general attitudes regarding genome sequencing

\begin{tabular}{|c|c|c|c|c|c|}
\hline & \multicolumn{5}{|l|}{ No. $(\%)^{\mathrm{a}}$} \\
\hline & $\begin{array}{l}\text { Strongly } \\
\text { disagree }\end{array}$ & $\begin{array}{l}\text { Somewhat } \\
\text { disagree }\end{array}$ & $\begin{array}{l}\text { Neither agree nor } \\
\text { disagree }\end{array}$ & $\begin{array}{l}\text { Somewhat } \\
\text { agree }\end{array}$ & $\begin{array}{l}\text { Strongly } \\
\text { agree }\end{array}$ \\
\hline \multicolumn{6}{|l|}{ Perceived utility of genome sequencing } \\
\hline I learned something to improve my health that I did not know before & $70(14.6)$ & $86(18.0)$ & $133(27.8)$ & $105(22.0)$ & $84(17.6)$ \\
\hline $\begin{array}{l}\text { Having personal genome sequencing made me feel like I have more } \\
\text { control over my health }\end{array}$ & $42(8.7)$ & $44(9.1)$ & $115(23.8)$ & $181(37.5)$ & $101(20.9)$ \\
\hline $\begin{array}{l}\text { What I learned from my personal genome sequencing will help reduce } \\
\text { my chances of getting sick }\end{array}$ & $83(17.2)$ & $104(21.6)$ & $175(36.3)$ & $78(16.2)$ & $42(8.7)$ \\
\hline $\begin{array}{l}\text { The information that I received about my genome will influence how I } \\
\text { manage my health in the future }\end{array}$ & $64(13.3)$ & $50(10.4)$ & $137(28.4)$ & $171(35.5)$ & $60(12.5)$ \\
\hline I am disappointed that my results did not tell me more information & $68(14.2)$ & $63(13.1)$ & $87(18.1)$ & $156(32.5)$ & $106(22.1)$ \\
\hline \multicolumn{6}{|l|}{ Attitudes regarding genome sequencing } \\
\hline $\begin{array}{l}\text { Personal genomic information should be part of a standard medical } \\
\text { record }\end{array}$ & $7(1.5)$ & $34(7.2)$ & $54(11.4)$ & $152(32.2)$ & $225(47.7)$ \\
\hline Health insurance should cover personal genome sequencing & $26(5.5)$ & $56(11.8)$ & $95(20.0)$ & $128(26.9)$ & $171(35.9)$ \\
\hline $\begin{array}{l}\text { Personal genome sequencing should only be available to people } \\
\text { through their doctor }\end{array}$ & $177(37.3)$ & $109(23.0)$ & $62(13.1)$ & $65(13.7)$ & $61(12.9)$ \\
\hline
\end{tabular}

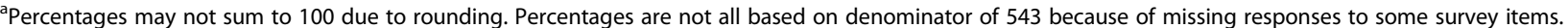
The percent of missing responses ranges between 11.0 and $13.1 \%$ (median $=11.8 \%$ missing)

\section{Discussion}

While an increasing number of healthy individuals are receiving PPGS results, the balance of benefits, harms, and downstream costs are still unclear. The PeopleSeq Consortium was formed to systematically collect short- and long-term medical, behavioral, and economic outcomes of PPGS. In this report, we have described the design and implementation of the PeopleSeq Consortium surveys, and the early data from four academic and industry PPGS projects.

Consistent with other PPGS studies, such as the NIH ClinSeq study [46], and with descriptions of users of DTC genetic testing [38, 47], most PeopleSeq participants are white and well-educated with high annual household incomes. Participants were motivated to undergo PPGS because of a general curiosity about their genetic make-up and disease risk, despite considering themselves to be healthy. Many of these participants had previously used DTC genetic testing for similar reasons. These early adopters were also highly knowledgeable about basic genomics concepts. Unsurprisingly, among individuals who all chose to undergo PPGS, concerns regarding privacy and insurance discrimination, which are often cited as serious deterrents [48, 49], were modest. Additionally, strong privacy and insurance discrimination concerns were lowest for PGP participants whose genomes were shared publicly, and these concerns were not relatively high for UYG participants whose clinical genome reports were sent directly to their physicians.

The PeopleSeq Consortium is a self-selected group of early adopters of genome sequencing for whom the perceived risks of PPGS were not necessarily a deterrent. Similarly, in the ClinSeq study, which enrolled middle-aged adults from the general population who consented to undergo genome sequencing in a clinical research setting, participants were found to be high in dispositional optimism and resilience, personality traits that could make these individuals more accepting of this new technology and their genomic information despite the psychological risks [46]. The risk-taking behavior and demographics of the PeopleSeq Consortium is characteristic of innovators and early adopters of a new technology, who are the first to try an innovation and often provide the evidence needed before adoption by the majority, as described by the Diffusion of Innovations Theory [33]. These characteristics may be most apparent among participants from PGP who were the least concerned about privacy and insurance discrimination despite their genomic information being made publicly available online as part of the PGP's unique approach to genomic research. [15] While individuals enrolled in PeopleSeq across all of the projects are clearly non-representative, the general population also appears highly receptive to learning about their genomic information. As part of focus groups conducted among a sample of Geisinger patient-participants in establishing the MyCode ${ }^{\circ}$ Community Health Initiative, it was reported that a majority of participants were in favor of the return of genomic results despite possible anxiety or lack of clinical actionability [21]. In a population-based sample of U.S. adults surveyed to inform the design of the PMI's All of Us Research Program, there was a high level of willingness to participate in the proposed study, and most respondents were interested in receiving their personal health information, including genetic 
information [50]. Despite the apparent enthusiasm from the general public, these responses may not translate into actual participation [51]. Thus, while the PeopleSeq Consortium may not be representative of the larger populations that may undergo PPGS in the future, information reported by these innovators and early adopters may provide insights that will inform future policies and potentially promote wider adoption of the technology. The outcomes reported by the current users of PPGS contribute to the evidence needed for (or against) the utility of sequencing and provide information on the medical resources that may be necessary as the use of sequencing expands both commercially and clinically.

The homogenous sociodemographic characteristics of these early adopters of genome sequencing, who are predominantly white, wealthy, and well-educated, are similar to the demographics of innovators and early adopters of many new technologies [33], but should still be considered. This Consortium did not provide sequencing but enrolled participants who independently sought genome sequencing through established programs, thus reflecting the lack of diversity that has long been acknowledged in the research and genomics community [52-54]. Limited representation can restrict the possible benefits of genomics in underrepresented ethnic and racial populations due to greater variant misclassification in these populations, perpetuating health disparities $[52,55,56]$. These findings reinforce the need for improved efforts for inclusivity in research and access to genome sequencing, which is an aim of the PMI's All of Us Research Program [57]. The early adopters in the PeopleSeq Consortium, who are pursuing sequencing in its current state, may provide the much needed data to address the concerns about PPGS, such as the costs of follow-up care, that may limit participation by underrepresented groups [54].

After a median of 8 months from disclosure of their PPGS results, only $13.5 \%$ of PeopleSeq participants reported making an additional appointment with a healthcare provider specifically because of their results, and this was similar across projects despite differences in the PPGS reports provided. Slightly more than $50 \%$ of participants, however, reported that they discussed their results with their healthcare provider, suggesting that most of these individuals were integrating this information into their regular care rather than making specific appointments to do so. These results are consistent with prior studies of the medical and behavioral impact of commercial DTC genetic testing of common polygenic conditions: the PGen Study found that 35\% of respondents reported sharing their results with a healthcare provider [58], and similar proportions were reported in other DTC genetic testing studies $[59,60]$. This is slightly lower than the $51.2 \%$ who reported discussing their results with a healthcare provider in the current study, and could be due to differences in the complexity and scope of results that may be returned with genome sequencing compared to genetic testing for common conditions or the role of healthcare providers in ordering sequencing in some of the collaborating projects. An even higher percentage of participants $(79.2 \%)$ from the YPO and MD/PhD Genome Projects reported discussing their results with a healthcare provider compared to the other projects, and this could be due to more encouragement from the study stuff to have these discussions [17]. While most providers were willing to discuss the PPGS results, longer follow-up will reveal if and how the results are integrated into longer-term care and clinical decision-making. We found some dissatisfaction in participants' discussions about their PPGS results with their provider, even for participants from UYG which sent clinical genome reports directly to the ordering providers. Prior research has revealed that non-geneticist physicians feel unprepared for sequencing, unsure of their genomic knowledge and ability to interpret results [61-63]. A major concern is that the complexities of genome sequencing could result in a substantial number of individuals being falsely identified as at-risk for disease [6, 7]. This could create distress or a fatalistic response on the individual level, iatrogenic harm from unnecessary surveillance or procedures, or overutilization of healthcare resources [9]. Alternatively, individuals without significant findings may misinterpret those negative results as an indication that they are at no risk, and could have a false sense of security and not seek appropriate medical care.

Participant-reported personal utility or value of PPGS was high, with the perceived utility in the future significantly outranking current utility. Most participants reported satisfaction with their sequencing experience, and very few reported any decisional regret. Additionally, almost a quarter of participants both strongly agreed that personal genomic information should be part of the medical record and strongly disagreed that sequencing should only be available through a doctor, indicating comfort with access to sequencing outside of the healthcare system, but a perceived utility of the results for their medical care. Despite high overall satisfaction with sequencing, more than half of participants were disappointed that their results did not provide them with more information, a finding also observed in clinical settings and with DTC genetic testing $[64,65]$. These high expectations of sequencing may reflect a lack of understanding of the current capabilities of this technology and the role of genetics (compared to lifestyle and environmental factors) on health. Though concerns have often focused on disclosure of unanticipated findings, these unrealistically high expectations of genomics should also be appropriately addressed in advance of disclosure of genomic results. 
While experts and professional societies recommend caution around incorporating PPGS into standard clinical practice as a screening tool, sequencing is more economically and logistically feasible, and commercial ventures are responding to public interest by providing both true DTC, consumer-facing physician mediated, and traditional provider-mediated genomic products. As interest in PPGS grows, the need to address the gaps in our understanding of the implications of PPGS in healthy individuals becomes more urgent. Results from the PeopleSeq Consortium thus far do not appear to support many of the current concerns regarding the negative consequences of PPGS. Participants reported feelings of empowerment, and very few reported any distress or regret after receiving their personal genomic information. The participants reported sharing their genomic results with their healthcare providers, and the Consortium continues to collect data on the medical responses to quantify the utility or disutility of sequencing.

The strengths of the PeopleSeq Consortium include its longitudinal design, which allows for the collection of both short- and long-term outcomes of PPGS, and the breadth of behavioral and medical data collected. Furthermore, these outcomes are being examined across both research and commercial PPGS projects that have not been previously explored in the literature. As the Consortium is a collaboration of multiple PPGS projects with different protocols, there is a heterogeneous sequencing experience; however, this captures the current access points of PPGS and enables the exploration of PPGS experiences across different approaches to the return of genomic results. These findings then provide a summary of the outcomes of sequencing across the currently available means by which individuals can pursue PPGS. There are limitations to the study. The response rate is currently below $50 \%$, but methods are being implemented to increase the proportion of responders moving forward. The results could be affected by nonresponder bias; however, the demographic differences observed between responders and nonresponders, while statistically significantly different, were not large and were similar to trends found in other studies of nonresponse [66]. Additionally, we currently do not have information on the individual genomic results that each participant received, and responses may vary depending on the content of the personal genomic reports. Thus, these findings are a summary of the responses and attitudes of individuals to PPGS results in general. Lastly, these results may not be generalizable, as early adopters of PPGS, like innovators and early adopters of most technologies, are different than the general public [33], notably being less diverse and potentially having greater risk-taking tendencies. Yet, little is currently known about the outcomes or attitudes of anyone undergoing
PPGS and receiving their personal genomic results, and these individuals are likely to be representative of the innovators and early adopters in our society who are shaping the use of genomic technologies and much can be learned from their experience.

The Consortium is continuing to add additional projects, enroll additional participants, and administer annual follow-up surveys beyond the initial results reported on here. As participants report sequencing-related healthcare use over time, the associated costs will also be estimated and evaluated. This will answer questions on the economic impact of sequencing in healthy individuals, given concerns regarding the possible burden of sequencing on the healthcare system. Furthermore, participants' genomic result reports are being collected by the Consortium, which will provide for rich analyses of outcomes as they relate to the actual genomic results the participants received. Additional strategies are being incorporated to improve the participant response rate and avoid attrition over longer-term follow-up.

\section{Conclusions}

Here we have reported on the design and implementation of the PeopleSeq Consortium, a collaboration collecting and examining the experiences and medical, behavioral, and economic outcomes of PPGS in healthy individuals. These healthy individuals who underwent predispositional sequencing were not deterred by concerns of privacy of their genomic information or possible insurance discrimination. Participants were enthusiastic about their experience and not distressed by their results. Many participants reported value in their health-related results, and approximately half reported discussing their results with a healthcare provider; though few participants reported making medical or lifestyle changes. The participants in the PeopleSeq Consortium are early adopters of PPGS and are providing novel information on the attitudes and outcomes of current users of PPGS.

\section{Additional files}

Additional file 1: Table S1. Overview of projects in the PeopleSeq Consortium included in this analysis. Table $\mathbf{S 2}$. Study measures by time point in the PeopleSeq Consortium included in this analysis. Figure S1. PeopleSeq Consortium enrollment and data collection by project.

Table S3. Reported psychological, behavioral, and medical reactions following disclosure of genome sequencing results by respondents to the post-disclosure survey and catch-up survey. Table S4. Degree of agreement/disagreement on perceived utility and general attitudes regarding genome sequencing by respondents to the post-disclosure survey and catch-up survey. Figure S2. Distributions of demographic characteristics by responders and nonresponders in a substudy of the PeopleSeq Consortium. Table S5. Characteristics of participants with completed post-disclosure or catch-up surveys in the PeopleSeq Consortium by project. (PDF, 631.7 KB). (PDF 631 kb) 
Additional file 2: The PeopleSeq Consortium surveys (pre-disclosure, post-disclosure, and catch-up surveys). (PDF, 1.4 MB). (PDF 1465 kb)

\section{Abbreviations}

CLIA: Clinical Laboratory Improvement Amendments; DTC: Direct-toconsumer; PeopleSeq: Personal Genome Sequencing Outcomes Consortium; PGen: Impact of Personal Genomics Study; PGP: Harvard Personal Genome Project; PMI: Precision Medicine Initiative; PPGS: Predispositional personal genome sequencing; UYG: Illumina's Understand Your Genome ; YPO: Baylor College of Medicine's Young Presidents' Organization Genome Project

\section{Acknowledgements}

In-kind support in the form of data collection, methodology, and data management services has been provided by SoundRocket.

Members of the PeopleSeq Consortium are as follows: Robert C. Green, Wendi Betting, Emilie Zoltick, Michael Linderman, Madeleine Ball, George Church, Scott D. Crawford, Ed Esplin, Eden Haverfield, Erica Ramos, Molly McGinniss, Debra Leonard, Amy McGuire, Thomas Caskey, Stacey Pereira, Saskia Sanderson, Thomas May, Candice Finnila, Brigette Davis, Kelly Hagman, Layla Shahmirzadi, Jane Juusola, Bethany Friedman, Stephanie DeWard, Ben Solomon, Bastian Greshake Tzovaras; and the investigators in the collaborating projects and institutes: Harvard's Personal Genome Project, Illumina's Understand Your Genome, Mount Sinai's HealthSeq Study, Baylor College of Medicine's CEO Genome Project, MD/PhD and YPO Genome Project, Invitae's Preventative Genetics Study, University of Vermont, Hudson Alpha, Ambry Genetics, GeneDx, OpenSNP, and SoundRocket.

\section{Funding}

This work was partially funded by an unrestricted grant from Illumina, Inc and is now supported by the NIH National Human Genome Research Institute grant R01-HG009922. ESZ is supported by the NIH National Heart, Lung, and Blood Institute grant T32-HL125232. MDL is partially supported by the NIH National Human Genome Research Institute grant R03-HG008809. GMC and the Harvard PGP are partially supported by the Open Humans Foundation. ALM is partially supported by the NIH National Human Genome Research Institute grant U01-HG006500. CTC and the YPO Genome Project were partially supported by the Cullen Foundation. DEN was supported by a Canadian Institutes of Health Research Postdoctoral Fellowship Award. RCG is also supported by NIH grants U01-HG006500, U19-HD077671, U01HG008685, and UG3-OD023156 along with funding from the Broad Institute and the Department of Defense. Funding was also provided by the Franca Sozzani Fund for Preventive Genomics.

\section{Availability of data and materials}

The dataset generated and analyzed for the current study is not publicly available as participants did not provide informed consented for broad sharing of the data collected and the dataset contains information that could compromise participant privacy. The dataset for the current study is available from the corresponding author on reasonable request.

\section{Authors' contributions}

ESZ, MDL, MAM, ER, MPB, SP, ALM, SCS, DEN, SDC, and RCG were responsible for the conception or design of the work. MDL, MAM, ER, MPB, GMC, DGBL, SP, CTC, SCS, EES, DEN, SDC, and RCG were responsible for the data collection. ESZ and $\mathrm{MDL}$ were responsible for the data analysis and interpretation. ESZ, MDL, and RCG drafted the article. All authors provided critical revisions to the manuscript and read and approved the final manuscript.

\section{Ethics approval and consent to participate}

The Partners HealthCare Human Research Committee and the Baylor College of Medicine Institutional Review Board approved the entire study. Informed consent was obtained electronically from participants. Our study conforms to the Declaration of Helsinki.

\section{Consent for publication}

Not applicable.

\section{Competing interests}

MAM is a paid employee and shareholder of Ilumina, Inc. ER is a former employee of Illumina, Inc., and is an advisor and shareholder of doc.ai.
GMC has advisory roles and research sponsorships from several companies (http://arep.med.harvard.edu/gmc/tech.html). EES is CEO of Sema4. SDC is the owner and CEO of SoundRocket, the company that conducted the data collection. RCG receives compensation for speaking or consultation from AIA, Helix, Ohana, Prudential, and Veritas, and is co-founder and advisor to Genome Medical, Inc. All other authors declare that they have no competing interests.

\section{Publisher's Note}

Springer Nature remains neutral with regard to jurisdictional claims in published maps and institutional affiliations.

\begin{abstract}
Author details
'Division of Genetics, Department of Medicine, Brigham and Women's Hospital, 41 Avenue Louis Pasteur, Suite 301, Boston, MA 02115, USA.

${ }^{2}$ Section of Preventive Medicine and Epidemiology, Department of Medicine, Boston University School of Medicine, 801 Massachusetts Avenue, Suite 470, Boston, MA 02118, USA. ${ }^{3}$ Department of Computer Science, Middlebury College, McCardell Bicentennial Hall, Middlebury, VT 05753, USA. ${ }^{4}$ Department of Genetics and Genomic Sciences, Icahn School of Medicine at Mount Sinai, One Gustave L. Levy Place, Box 1498, New York, NY 10029, USA. ${ }^{5}$ lllumina, Inc, 5200 Illumina Way, San Diego, CA 92122, USA. ${ }^{6}$ Geisinger National Precision Health, Geisinger, 6101 Executive Blvd, Suite 110, North Bethesda, MD 20852, USA. ${ }^{7}$ Open Humans Foundation, Boston, MA, USA. ${ }^{8}$ Harvard Personal Genome Project, Harvard Medical School, Boston, MA, USA. ${ }^{9}$ Department of Genetics, Harvard Medical School, 77 Avenue Louis Pasteur, Room 238, Boston, MA 02115, USA. ${ }^{10}$ Wyss Institute for Biologically Inspired Engineering, Harvard University, 3 Blackfan Circle, Boston, MA 02115, USA. ${ }^{11}$ Department of Pathology and Laboratory Medicine, Robert Larner, M.D, College of Medicine of the University of Vermont, 89 Beaumont Avenue, Courtyard at Given S269, Burlington, VT 05405, USA. ${ }^{12}$ Center for Medical Ethics and Health Policy, Baylor College of Medicine, One Baylor Plaza, Suite 310D, Houston, TX 77030, USA. ${ }^{13}$ Molecular and Human Genetics, Baylor College of Medicine, One Baylor Plaza, Mail Stop BCM225, Houston, TX 77030, USA. ${ }^{14}$ Department of Behavioural Science and Health, University College London, Gower Street, London WC1E 6BT, UK. ${ }^{15}$ School of Human Nutrition, McGill University, 21111 Lakeshore Road, Ste-Anne-de-Bellevue, Quebec H9X 3V9, Canada. ${ }^{16}$ SoundRocket, 950 Victors Way, Suite 50, Ann Arbor, MI 48108, USA. ${ }^{17}$ The Broad Institute of MIT and Harvard, 415 Main Street, Cambridge, MA 02142, USA. ${ }^{18}$ Harvard Medical School, Boston, MA, USA. ${ }^{19}$ Partners HealthCare Personalized Medicine, Boston, MA 02115, USA.
\end{abstract}

Received: 29 August 2018 Accepted: 5 February 2019

Published online: 27 February 2019

\section{References}

1. Biesecker $L G$, Green RC. Diagnostic clinical genome and exome sequencing. N Engl J Med. 2014;370(25):2418-25.

2. Green RC, Rehm HL, Kohane IS. Clinical genome sequencing. In: Ginsburg GS, Willard HF, editors. Genomic and personalized medicine. 2nd ed. San Diego: Academic Press; 2013. p. 102-22.

3. Sabour L, Sabour M, Ghorbian S. Clinical applications of next-generation sequencing in cancer diagnosis. Pathol Oncol Res. 2017;23(2):225-34.

4. Linderman MD, Nielsen DE, Green RC. Personal genome sequencing in ostensibly healthy individuals and the PeopleSeq Consortium. J Pers Med. 2016;6(2):14

5. Rehm HL. Evolving health care through personal genomics. Nat Rev Genet. 2017;18(4):259-67.

6. Adams MC, Evans JP, Henderson GE, Berg JS. The promise and peril of genomic screening in the general population. Genet Med. 2016;18(6):593-9.

7. Khoury MJ, McCabe LL, McCabe ER. Population screening in the age of genomic medicine. N Engl J Med. 2003;348(1):50-8.

8. Cooper DN, Krawczak M, Polychronakos C, Tyler-Smith C, Kehrer-Sawatzki H. Where genotype is not predictive of phenotype: towards an understanding of the molecular basis of reduced penetrance in human inherited disease. Hum Genet. 2013;132(10):1077-130.

9. Christensen KD, Dukhovny D, Siebert U, Green RC. Assessing the costs and cost-effectiveness of genomic sequencing. J Pers Med. 2015;5(4):470-86.

10. Abul-Husn NS, Manickam K, Jones LK, Wright EA, Hartzel DN, GonzagaJauregui C, et al. Genetic identification of familial hypercholesterolemia 
within a single U.S. health care system. Science. 2016;354(6319):aaf7000. https://doi.org/10.1126/science.aaf7000.

11. Perkins BA, Caskey CT, Brar P, Dec E, Karow DS, Kahn AM, et al. Precision medicine screening using whole-genome sequencing and advanced imaging to identify disease risk in adults. Proc Natl Acad Sci U S A. 2018; 115(14):3686-91.

12. Dewey FE, Murray MF, Overton JD, Habegger L, Leader JB, Fetterolf SN, et al. Distribution and clinical impact of functional variants in 50,726 wholeexome sequences from the DiscovEHR study. Science. 2016;354(6319): aaf6814. https://doi.org/10.1126/science.aaf6814.

13. Vassy JL, Christensen KD, Schonman EF, Blout CL, Robinson JO, Krier JB, et al. The impact of whole-genome sequencing on the primary care and outcomes of healthy adult patients: a pilot randomized trial. Ann Intern Med. 2017;167(3):159-69.

14. Johnston JJ, Lewis KL, Ng D, Singh LN, Wynter J, Brewer C, et al. Individualized iterative phenotyping for genome-wide analysis of loss-offunction mutations. Am J Hum Genet. 2015;96(6):913-25.

15. Lunshof JE, Bobe J, Aach J, Angrist M, Thakuria JV, Vorhaus DB, et al. Personal genomes in progress: from the human genome project to the personal genome project. Dialogues Clin Neurosci. 2010;12(1):47-60.

16. Biesecker LG, Mullikin JC, Facio FM, Turner C, Cherukuri PF, Blakesley RW, et al. The ClinSeq Project: piloting large-scale genome sequencing for research in genomic medicine. Genome Res. 2009;19(9):1665-74.

17. Gonzalez-Garay ML, McGuire AL, Pereira S, Caskey CT. Personalized genomic disease risk of volunteers. Proc Natl Acad Sci U S A. 2013;110(42):16957-62.

18. Vassy JL, Lautenbach DM, McLaughlin HM, Kong SW, Christensen KD, Krier J, et al. The MedSeq Project: a randomized trial of integrating whole genome sequencing into clinical medicine. Trials. 2014;15:85.

19. Sanderson SC, Linderman MD, Suckiel SA, Zinberg R, Wasserstein M, Kasarskis A, et al. Psychological and behavioural impact of returning personal results from whole-genome sequencing: the HealthSeq project. Eur J Hum Genet. 2017;25(3):280-92.

20. Lindor NM, Schahl KA, Johnson KJ, Hunt KS, Mensink KA, Wieben ED, et al. Whole-exome sequencing of 10 scientists: evaluation of the process and outcomes. Mayo Clin Proc. 2015;90(10):1327-37.

21. Carey DJ, Fetterolf SN, Davis FD, Faucett WA, Kirchner HL, Mirshahi U, et al. The Geisinger MyCode community health initiative: an electronic health record-linked biobank for precision medicine research. Genet Med. 2016; 18(9):906-13.

22. Kauffman TL, Wilfond BS, Jarvik GP, Leo MC, Lynch FL, Reiss JA, et al. Design of a randomized controlled trial for genomic carrier screening in healthy patients seeking preconception genetic testing. Contemp Clin Trials. 2017;53:100-5.

23. Holm IA, Agrawal PB, Ceyhan-Birsoy O, Christensen KD, Fayer S, Frankel LA, et al. The BabySeq project: implementing genomic sequencing in newborns. BMC Pediatr. 2018;18(1):225.

24. Collins FS, Varmus H. A new initiative on precision medicine. N Engl I Med. 2015;372(9):793-5.

25. Ambry Genetics. http://www.ambrygen.com/. Accessed 20 Aug 2018.

26. GeneDx. http://www.genedx.com/. Accessed 20 Aug 2018.

27. Invitae. https:/www.invitae.com/. Accessed 20 Aug 2018.

28. Genos. https://genos.co/. Accessed 20 Aug 2018.

29. Arivale. https://www.arivale.com/. Accessed 20 Aug 2018.

30. Color. https://www.color.com/. Accessed 20 Aug 2018.

31. Helix. https://www.helix.com/. Accessed 20 Aug 2018.

32. Human Longevity, Inc. http://www.humanlongevity.com/. Accessed 20 Aug 2018.

33. Rogers ME. Diffusion of innovations. 5th ed. New York: Free Press; 2003.

34. Choe EK, Lee NB, Bongshin L, Pratt W, Kientz JA. Understanding quantifiedselfers' practices in collecting and exploring personal data. Proceedings of the SIGCHI Conference on Human Factors in Computing Systems. New York: ACM; 2014. p. 1143-1152.

35. Understand Your Genome. https://www.understandyourgenome.com/. Accessed 20 Aug 2018

36. Green RC, Berg JS, Grody WW, Kalia SS, Korf BR, Martin CL, et al. ACMG recommendations for reporting of incidental findings in clinical exome and genome sequencing. Genet Med. 2013;15(7):565-74.

37. Kalia SS, Adelman K, Bale SJ, Chung WK, Eng C, Evans JP, et al. Recommendations for reporting of secondary findings in clinical exome and genome sequencing, 2016 update (ACMG SF v2.0): a policy statement of the American College of Medical Genetics and Genomics. Genet Med. 2017;19(2):249-55
38. Carere DA, Couper MP, Crawford SD, Kalia SS, Duggan JR, Moreno TA, et al. Design, methods, and participant characteristics of the Impact of Personal Genomics (PGen) Study, a prospective cohort study of direct-to-consumer personal genomic testing customers. Genome Med. 2014;6(12):96.

39. Kroenke K, Spitzer RL, Williams JB, Monahan PO, Lowe B. Anxiety disorders in primary care: prevalence, impairment, comorbidity, and detection. Ann Intern Med. 2007;146(5):317-25.

40. Lowe B, Kroenke K, Grafe K. Detecting and monitoring depression with a two-item questionnaire (PHQ-2). J Psychosom Res. 2005;58(2):163-71.

41. Carleton RN, Norton MA, Asmundson GJ. Fearing the unknown: a short version of the Intolerance of Uncertainty Scale. J Anxiety Disord. 2007;21 (1):105-17.

42. Brehaut JC, O'Connor AM, Wood TJ, Hack TF, Siminoff L, Gordon E, et al. Validation of a decision regret scale. Med Decis Mak. 2003;23(4):281-92.

43. Kaphingst KA, McBride CM, Wade C, Alford SH, Reid R, Larson E, et al. Patients' understanding of and responses to multiplex genetic susceptibility test results. Genet Med. 2012;14(7):681-7.

44. Kaphingst KA, Facio FM, Cheng MR, Brooks S, Eidem H, Linn A, et al. Effects of informed consent for individual genome sequencing on relevant knowledge. Clin Genet. 2012;82(5):408-15.

45. McGuire AL, Diaz CM, Wang T, Hilsenbeck SG. Social networkers' attitudes toward direct-to-consumer personal genome testing. Am J Bioeth. 2009; 9(6-7):3-10

46. Lewis KL, Han PK, Hooker GW, Klein WM, Biesecker LG, Biesecker BB. Characterizing participants in the ClinSeq Genome Sequencing Cohort as early adopters of a new health technology. PLoS One. 2015;10(7):e0132690.

47. Bloss CS, Ornowski L, Silver E, Cargill M, Vanier V, Schork NJ, et al. Consumer perceptions of direct-to-consumer personalized genomic risk assessments. Genet Med. 2010;12(9):556-66.

48. Green RC, Lautenbach D, McGuire AL. GINA, genetic discrimination, and genomic medicine. N Engl J Med. 2015;372(5):397-9.

49. Robinson JO, Carroll TM, Feuerman LZ, Perry DL, Hoffman-Andrews L, Walsh RC, et al. Participants and study decliners' perspectives about the risks of participating in a clinical trial of whole genome sequencing. J Empir Res Hum Res Ethics. 2016;11(1):21-30.

50. Kaufman DJ, Baker R, Milner LC, Devaney S, Hudson KL. A survey of U.S adults' opinions about conduct of a nationwide Precision Medicine Initiative cohort study of genes and environment. PLoS One. 2016;11(8):e0160461.

51. Ropka ME, Wenzel J, Phillips EK, Siadaty M, Philbrick JT. Uptake rates for breast cancer genetic testing: a systematic review. Cancer Epidemiol Biomark Prev. 2006;15(5):840-55.

52. Rotimi CN. Health disparities in the genomic era: the case for diversifying ethnic representation. Genome Med. 2012;4(8):65.

53. Oh SS, Galanter J, Thakur N, Pino-Yanes M, Barcelo NE, White MJ, et al. Diversity in clinical and biomedical research: a promise yet to be fulfilled. PLoS Med. 2015;12(12):e1001918.

54. George S, Duran N, Norris K. A systematic review of barriers and facilitators to minority research participation among African Americans, Latinos, Asian Americans, and Pacific Islanders. Am J Public Health. 2014;104(2):e16-31.

55. Manrai AK, Funke BH, Rehm HL, Olesen MS, Maron BA, Szolovits P, et al. Genetic misdiagnoses and the potential for health disparities. N Engl J Med. 2016;375(7):655-65.

56. Caswell-Jin JL, Gupta T, Hall E, Petrovchich IM, Mills MA, Kingham KE, et al. Racial/ethnic differences in multiple-gene sequencing results for hereditary cancer risk. Genet Med. 2017;20(2):234-9.

57. Precision Medicine Initiative (PMI) Working Group. The Precision Medicine Initiative Cohort Program - Building a Research Foundation for 21st Century Medicine. 2015. https:/www.nih.gov/sites/default/files/research-training/ initiatives/pmi/pmi-working-group-report-20150917-2.pdf. Accessed 20 Aug 2018.

58. van der Wouden $\mathrm{CH}$, Carere DA, Maitland-van der Zee AH, MTt R, Roberts JS, Green RC, et al. Consumer perceptions of interactions with primary care providers after direct-to-consumer personal genomic testing. Ann Intern Med. 2016;164(8):513-22.

59. Kaufman DJ, Bollinger JM, Dvoskin RL, Scott JA. Risky business: risk perception and the use of medical services among customers of DTC personal genetic testing. J Genet Couns. 2012;21 (3):413-22.

60. Darst BF, Madlensky L, Schork NJ, Topol EJ, Bloss CS. Characteristics of genomic test consumers who spontaneously share results with their health care provider. Health Commun. 2014;29(1):105-8.

61. Christensen KD, Vassy JL, Jamal L, Lehmann LS, Slashinski MJ, Perry DL, et al. Are physicians prepared for whole genome sequencing? A qualitative analysis. Clin Genet. 2016;89(2):228-34. 
62. Selkirk CG, Weissman SM, Anderson A, Hulick PJ. Physicians' preparedness for integration of genomic and pharmacogenetic testing into practice within a major healthcare system. Genet Test Mol Biomarkers. 2013;17(3):219-25.

63. Mikat-Stevens NA, Larson IA, Tarini BA. Primary-care providers' perceived barriers to integration of genetics services: a systematic review of the literature. Genet Med. 2015;17(3):169-76.

64. Miller FA, Hayeems RZ, Bytautas JP, Bedard PL, Ernst S, Hirte H, et al. Testing personalized medicine: patient and physician expectations of nextgeneration genomic sequencing in late-stage cancer care. Eur J Hum Genet. 2014;22(3):391-5.

65. Roberts JS, Gornick MC, Carere DA, UhImann WR, Ruffin MT, Green RC. Direct-to-consumer genetic testing: user motivations, decision making, and perceived utility of results. Public Health Genomics. 2017;20(1):36-45.

66. Fan $W$, Yan Z. Factors affecting response rates of the web survey: a systematic review. Comput Hum Behav. 2010;26(2):132-9.

Ready to submit your research? Choose BMC and benefit from:

- fast, convenient online submission

- thorough peer review by experienced researchers in your field

- rapid publication on acceptance

- support for research data, including large and complex data types

- gold Open Access which fosters wider collaboration and increased citations

- maximum visibility for your research: over $100 \mathrm{M}$ website views per year

At $\mathrm{BMC}$, research is always in progress.

Learn more biomedcentral.com/submissions 